\title{
Caracterización de la intoxicación ocupacional por pesticidas en trabajadores agrícolas atendidos en el Hospital Barranca Cajatambo 2008 - 2017
}

Lincol Marx Cruz Aquino* 1,a; Maritza Dorila Placencia Medina 2,b

RESUMEN

Objetivo: Determinar las características de la intoxicación ocupacional por pesticidas en trabajadores agrícolas atendidos en el Hospital de Barranca 2008-2017.

Materiales y métodos: Estudio descriptivo, retrospectivo y transversal. Se estudiaron a 295 trabajadores agrícolas con intoxicación ocupacional por pesticidas atendidos en el Hospital de Barranca entre 2008 y 2017. Se incluyó a todo trabajador agrícola mayor de 14 años, hombre o mujer, con historia clínica completa de intoxicación por pesticidas. Las variables fueron características sociodemográficas, epidemiológicas, del pesticida y de la intoxicación. Se emplearon frecuencias absolutas $(n)$ para las variables cualitativas y frecuencias relativas (\%) para las variables relativas. Para las variables cuantitativas se emplearon medidas de tendencia central (promedio) y de dispersión (desviación estándar). Los datos se procesaron en el programa SPSS vs 25 y se presentaron en tablas simples.

Resultados: El 98 \% de los participantes fueron hombres, entre 15 a 25 años $(47,5 \%)$, solteros $(53,6 \%)$, con educación secundaria $(68,1 \%)$, aplicadores de plaguicidas $(67,5 \%)$, con IMC normal $(56,9 \%$ ) y sin comorbilidades $(97,6 \%)$. Los pesticidas fueron fungicidas $(39,7 \%)$ y herbicidas $(37,6 \%)$, de tipo organofosforados $(98,0 \%)$ y medianamente tóxicos $(96,3 \%)$. La vía de ingreso al organismo fue respiratoria (57,3\%) y piel (42,0\%). En relación a la intoxicación, el tiempo entre exposición y momento en que acude al hospital fue entre 1 a 3 horas (53,9\%); la severidad de intoxicación fue moderada $(94,2 \%)$, las manifestaciones clínicas más frecuentes fueron vómito (79 \%) y náuseas $(72,5 \%)$. El tratamiento se realizó con solución IV $(99,7 \%)$ y atropina $(81,7 \%)$. El 99,3\% de los casos evolucionó sin complicaciones intrahospitalarias. Finalmente, el 100 \% de los pacientes salió del hospital en estado de mejoría.

Conclusiones: La mayoría de los trabajadores agrícolas intoxicados por pesticidas, son jóvenes de sexo masculino, solteros, con instrucción secundaria, que aplican plaguicidas, sin comorbilidades y con IMC normal. Los pesticidas más usados fueron fungicidas y herbicidas, de mediana categoría tóxica, con vía de ingreso respiratoria. La intoxicación fue moderada, sin complicaciones intrahospitalarias. Nuestro estudio evalúa los efectos a corto plazo de la intoxicación por pesticidas en la producción agrícola, es necesario considerar efectos a largo plazo como cáncer y daño neurológico. Al respecto, el Gobierno debe revisar la política relacionada al tema para reducir la producción y aplicación de pesticidas.

Palabras clave: Medicina del trabajo; Exposición a plaguicidas; Agricultores (Fuente: DeCS BIREME).

\section{Characteristics of occupational pesticide poisoning in agricultural workers treated at the Hospital Barranca Cajatambo during 2008 - 2017}

\section{ABSTRACT}

Objective: To determine the characteristics of occupational pesticide poisoning in agricultural workers treated at the Hospital Barranca Cajatambo during 2008-2017.

Materials and methods: A descriptive, retrospective and cross-sectional study conducted in a population of 295 agricultural workers poisoned by occupational pesticide exposure and treated at the Hospital Barranca Cajatambo from 2008 to 2017. Every male or female agricultural worker aged over 14 with a complete medical record of pesticide poisoning was included in the study. The variables were the sociodemographic and epidemiological characteristics of the pesticide and intoxication. Absolute frequencies $(n)$ were used for the qualitative variables and relative frequencies (\%) for the relative variables. Measures of central tendency (average) and dispersion tendency (standard deviation) were used for the quantitative variables. Data was processed using software SPSS v25.0 and presented in simple tables. 
Results: Ninety-eight percent (98.0\%) of the study subjects were males, $47.5 \%$ were between 15 and 25 years, $53.6 \%$ were single, $68.1 \%$ had secondary education, $67.5 \%$ worked as pesticide applicators, $56.9 \%$ had a normal $\mathrm{BMI}$, and $97.6 \%$ presented no comorbidities. Pesticides were classified as fungicide $(39.7 \%)$, herbicide (37.6\%), organophosphorus $(98.0 \%)$ and moderately toxic $(96.3 \%)$. The routes of entry to the body were the airways $(57.3 \%)$ and skin $(42.0 \%)$. In relation to poisoning, the time between pesticide exposure and arrival to the hospital ranged from 1 to 3 hours $(53.9 \%)$. The severity of poisoning was moderate $(94.2 \%)$. The most frequent clinical manifestations were vomiting (79\%) and nausea (72.5\%). Treatment included IV medication (99.7\%) and atropine $(81.7 \%)$. Ninety-nine point three percent $(99.3 \%)$ of the study population progressed with no hospitalacquired complications. Finally, $100 \%$ of the patients improved their health condition at discharge.

Conclusions: Most agricultural workers poisoned by pesticides were young, male and single; had secondary education; worked as pesticide applicators; did not present comorbidities; and had a normal BMI. The most frequently used pesticides were fungicides, herbicides, those moderately toxic and those entering the body through the airways. Poisoning was moderate with no hospital-acquired complications. The present study evaluates the short-term effects of pesticide poisoning in agricultural production. It is necessary to take into account its long-term effects such as cancer and neurological conditions. In this regard, the government should review the policy related to reducing the production and application of pesticides.

Keywords: Occupational medicine; Pesticide exposure; Farmers (Source: MeSH NLM).

1. Hospital Barranca Cajatambo SBS. Barranca, Perú.

2. Universidad Nacional Mayor de San Marcos, Facultad de Medicina, Departamento de Ciencias Dinámicas. Lima, Perú.

a. Médico Cirujano.

b. Doctora en Farmacia y Bioquímica.

* Autor corresponsal. 


\section{INTRODUCCIÓN}

Los pesticidas o plaguicidas han sido utilizados ampliamente para controlar las plagas en la agricultura, y es una práctica beneficiosa para la producción de alimentos a nivel mundial con un aumento notable en el rendimiento de la misma ${ }^{(1)}$. Aunque los pesticidas se han desarrollado para funcionar con una certeza razonable y un riesgo mínimo para la salud humana y el medio ambiente, esta práctica no está exenta de causar efectos dañinos en la salud de las personas que trabajan directa y frecuentemente con pesticidas ${ }^{(2)}$. Debido a su reactividad biológica inherente, los pesticidas son potencialmente peligrosos para la salud humana. La intoxicación por pesticidas a consecuencia del trabajo agrícola ha sido reconocida como un importante problema de salud pública entre los agricultores de todo el mundo. Según un último informe de las Naciones Unidas, a nivel mundial, alrededor de 200000 muertes por año son el resultado de envenenamiento agudo por plaguicidas, y cerca del $99 \%$ de estas muertes se produjeron en países en desarrollo donde las reglamentaciones de salud, seguridad y medio ambiente eran más débiles ${ }^{(3)}$. Además, las intoxicaciones agudas por pesticidas afectan con preferencia a varones jóvenes, que son los que suelen estar en contacto en el ámbito agrícola con estos tóxicos. Plazas y Olarte (4) refieren que, en general, las intoxicaciones son más frecuentes en niños, agricultores y trabajadores de manufactura (floricultura, ganaderos, fumigadores). Estos autores también indican que las causas de la mortalidad son variadas e incluyen alta toxicidad de los productos, largos desplazamientos desde el área rural a centros de alta complejidad para un adecuado manejo y el desconocimiento por parte del personal de salud del manejo estándar y el tratamiento especial con antídotos. En Latinoamérica, la exposición a plaguicidas constituye un problema de salud, y la población femenina es la más afectada. En países como Colombia, Chile, Perú y Costa Rica desde hace 15 años se ha demostrado un alto índice de exposición de plaguicidas en mujeres en edad fértil, lo que involucra un riesgo a la salud en general y más aún sobre la reproducción ${ }^{(5)}$. En Paraguay, los plaguicidas son el agente causal del $13,7 \%$ de las intoxicaciones reportadas, y se sabe que, por cada 15 casos de intoxicación aguda, 10 se reportan en mujeres y 5 en varones, todos ellos con un rango de edad entre los 5 y los 67 años de edad (6). En Colombia, debido a la alta producción agrícola y una frontera agrícola en expansión, los plaguicidas son los mayores causantes de intoxicaciones en toda la región. Se calcula que tienen una incidencia del $68 \%$ y son responsables de la mortalidad en un $66 \%{ }^{(7)}$.

En el Perú, el sector agrícola es uno de las principales áreas productivas del país, y cuenta, desde hace pocos años, con una Norma Técnica de Salud, la de Vigilancia epidemiológica del riesgo de exposición e intoxicación por plaguicidas, que tiene como objetivo prevenir y controlar la exposición e intoxicación por pesticidas en quienes se dedican a las labores relacionadas directamente con la agricultura y a toda la población.

El Hospital de Barranca pertenece a la jurisdicción de la Red de Salud Barranca-Cajatambo, que abarca las provincias de Barranca y de Cajatambo; tiene asignados un total de 151355 habitantes. Asimismo, Barranca tiene como actividad económica principal a la agricultura que cubre el 77,7\% de los ingresos familiares. Esta actividad se desarrolla tanto en empresas como en fundos particulares por lo que es necesario conocer el manejo de pacientes con intoxicación aguda por pesticidas, principalmente, si se debe a exposición a los de tipo organofosforados (O-P). Además, se observan dos situaciones alarmantes, una de ellas es que la fumigación de los campos se realiza sin el Equipo de Protección Personal (EPP); la segunda, que tanto las empresas informales como los fundos particulares emplean a adolescentes para la fumigación con O-P, lo que los expone a diversos problemas de salud y mayores riesgos para su vida.

El presente estudio busca determinar las características de la intoxicación ocupacional por pesticidas en trabajadores agrícolas atendidos en el Hospital de Barranca-Cajatambo en 2008-2017. Así, el personal sanitario adquiere nuevos conocimientos o actualiza los que ya existen para brindar atención personalizada y de calidad a los usuarios (pacientes) que acuden al nosocomio donde se realiza el estudio. Esto permitirá realizar oportunamente las acciones necesarias para evitar complicaciones que pueden afectar la vida de los pacientes; y además permitirá que los trabajadores agrícolas tomen las medidas de seguridad necesarias para evitar dicho problema, que es perjudicial para su vida, puesto que los afecta en diversos ámbitos como en lo personal, social, laboral y familiar.

El uso de pesticidas en la agricultura puede ocasionar diferentes afecciones en la salud de las personas que realizan esta actividad, más aún cuando las condiciones (medidas de bioseguridad) y medios de trabajo (tecnología) no son los adecuados. Los trabajadores agrícolas de las provincias de Barranca y Cajatambo que sufren intoxicación aguda por pesticidas son trasladados de emergencia a los establecimientos de salud de la Red Barranca-Cajatambo para su oportuna atención y referencia, según el caso, al Hospital Barranca. Al momento de realizarse la atención por emergencia y administrar la terapia farmacológica según el protocolo establecido, se procede al registro epidemiológico y llenado de la Ficha Única de Aviso de Accidente de Trabajo. 


\section{MATERIALES Y MÉTODOS}

Diseño y población de estudio

Estudio descriptivo, retrospectivo y transversal. La población estuvo conformada por 295 casos de trabajadores agrícolas intoxicados por pesticidas de origen ocupacional, atendidos en el Hospital Barranca en el periodo de 2008 a 2017. El tipo de muestreo fue por conveniencia y la técnica de muestreo fue de todos los casos. Los criterios de inclusión fueron que la persona sea un trabajador agrícola intoxicado por pesticidas en el periodo 2008-2017, hombre o mujer, mayor de 14 años de edad, que haya sido atendido en el Hospital de Barranca y que tenga historia clínica completa de la intoxicación por pesticidas.

\section{Variables y mediciones}

Las variables fueron las características sociodemográficas, las epidemiológicas, las del pesticida y las relacionadas a la intoxicación. El instrumento de medición fue la ficha de recolección de datos que se dividió en cuatro partes para las variables empleadas.

\section{Análisis estadístico}

Para las variables cualitativas se emplearon frecuencias absolutas ( $n$ ) y relativas (\%); mientras que en las variables cuantitativas se usó medidas de tendencia central (promedio) y de dispersión (desviación estándar). Los datos fueron procesados por el programa SPSS vs 25 , se presentan en tablas simples.

\section{RESULTADOS}

Se encontraron 295 trabajadores agrícolas intoxicados por pesticidas. La edad promedio fue de $31,07 \pm 14,549$ (15-83), 98 \% eran hombres, y la mayoría de ellos era soltero $(53,6 \%)$. El grado de instrucción predominante fue la secundaria $(68,1 \%)$ y el puesto de trabajo más frecuente fue aplicador de plaguicidas $(67,5 \%)$.

En la figura 1 se observa el histograma de frecuencia del índice de masa corporal (IMC), donde el mayor porcentaje $(76,3 \%)$ se encuentra entre los rangos 20 y 30 .

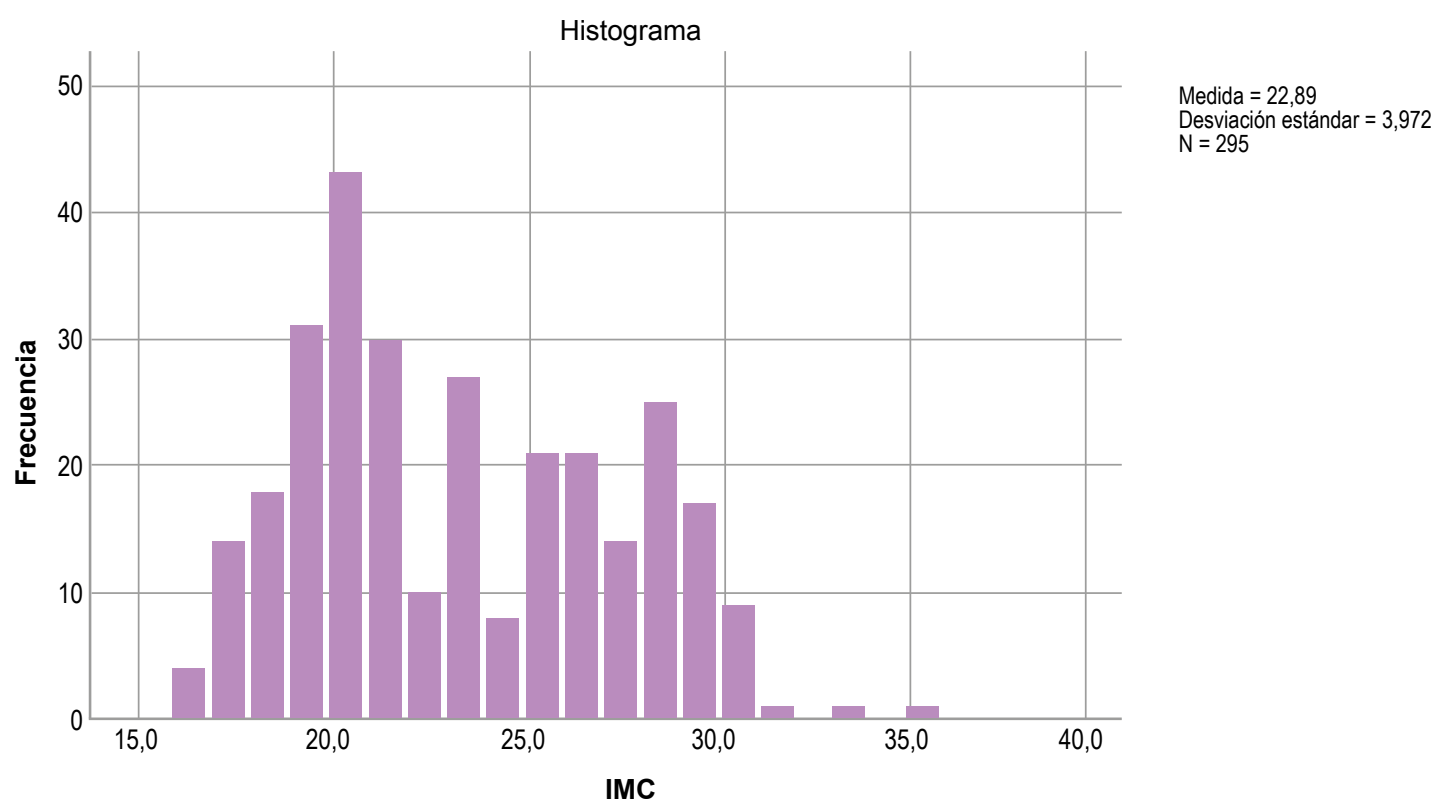

Figura 1. Histograma de frecuencia del índice de masa corporal (IMC) de los trabajadores agrícolas atendidos en el Hospital de Barranca 2008-2017

En la tabla 1 se observa que el $1,7 \%$ de los trabajadores intoxicados por pesticidas tenían como comorbilidad la hipertensión arterial, seguido de diabetes mellitus $(0,3 \%)$ e insuficiencia renal $(0,3 \%)$. El índice de masa corporal predominante entre los trabajadores intoxicados fue normal (56,9\%), mientras que $32,9 \%$ presentó sobrepeso y $6,1 \%$, y bajo peso. 
Tabla 1. Características epidemiológicas de los trabajadores agrícolas intoxicados por pesticidas atendidos en el Hospital de Barranca 2008-2017

\begin{tabular}{|lcc|}
\hline $\begin{array}{l}\text { Características } \\
\text { epidemiológicas }\end{array}$ & $\mathbf{N}$ & $\%$ \\
\hline Comorbilidades & 5 & \\
\hline Hipertensión arterial & 1 & 1,7 \\
\hline Diabetes mellitus & 1 & 0,3 \\
\hline Insuficiencia renal & 288 & 0,3 \\
\hline Ninguno & 18 & 97,6 \\
\hline IMC & 168 & 6,1 \\
\hline Bajo peso & 97 & 56,9 \\
\hline Normal & 11 & 32,9 \\
\hline Sobrepeso & 1 & 3,7 \\
\hline Obesidad de tipo I & 295 & 0,3 \\
\hline Obesidad de tipo II & & 100 \\
\hline$\quad$ Total & & \\
\hline
\end{tabular}

En la figura 2 se observa que el $39,7 \%$ de los trabajadores agrícolas se intoxicaron con fungicidas, el $37,6 \%$ con herbicidas, el $4,1 \%$ con fumigantes, y solo el $0,3 \%$ con rodenticidas.

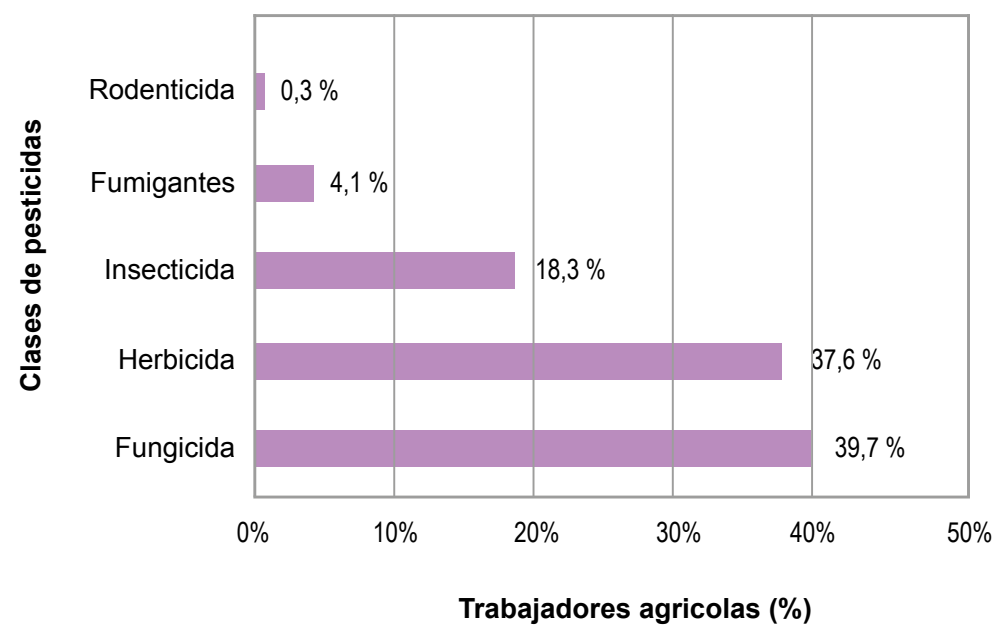

Figura 2. Clase de pesticidas que intoxicaron a los trabajadores agrícolas atendidos en el Hospital de Barranca 2008-2017

La figura 3 presenta la distribución según categoría tóxica, donde el $96,3 \%$ fue afectado por un pesticida de categoría III (medianamente tóxicos), el 3,1 \% por uno de categoría IV (ligeramente tóxicos), el 0,3\% por uno de categoría II (altamente tóxicos), y otro 0,3 \% por la categoría más alta, (I, extremadamente tóxicos). 
Caracterización de la intoxicación ocupacional por pesticidas en trabajadores agrícolas atendidos en el Hospital Barranca Cajatambo 2008 - 2017

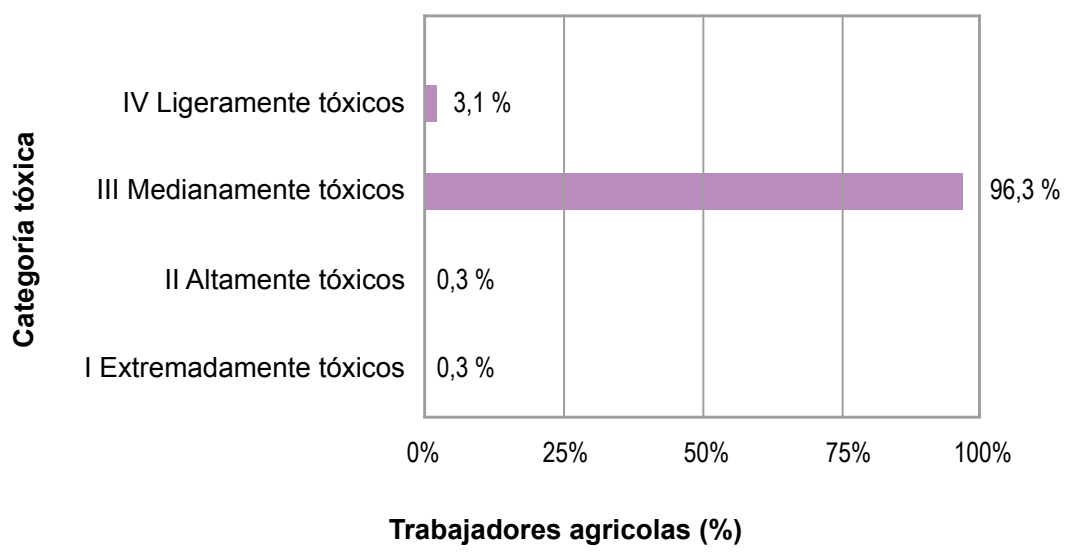

Figura 3. Categoría tóxica de los pesticidas que intoxicaron a los trabajadores agrícolas atendidos en el Hospital de Barranca 2008-2017

La tabla 2 muestra las características de los 57,3\% ingresaban al organismo por medio de las vías pesticidas, el $98,0 \%$ eran organofosforados y el respiratorias.

Tabla 2. Características del pesticida que intoxicó a los trabajadores agrícolas atendidos en el Hospital de Barranca $2008-2017$

\begin{tabular}{|c|c|c|}
\hline $\begin{array}{l}\text { Características del } \\
\text { pesticida }\end{array}$ & $\mathbf{N}$ & $\%$ \\
\hline \multicolumn{3}{|l|}{ Tipo de pesticida } \\
\hline Organofosforados & 289 & 98,0 \\
\hline Carbamatos & 6 & 2,0 \\
\hline \multicolumn{3}{|c|}{ Vías de ingreso al organismo } \\
\hline Vías respiratorias & 169 & 57,3 \\
\hline Piel & 124 & 42,0 \\
\hline Mucosas & 1 & 0,3 \\
\hline Oral & 1 & 0,3 \\
\hline Total & 295 & 100 \\
\hline
\end{tabular}

La tabla 3 muestra las características relacionadas a la intoxicación de los trabajadores agrícolas. El 53,2 \% tenía entre 1 a 3 horas de exposición hasta que acudió al hospital; el 94,2 \% tenía intoxicación de severidad moderada. Las manifestaciones clínicas más frecuentes fueron los vómitos (79,0 \%) y las náuseas (72,5\%). Respecto al tratamiento médico, 99,7 \% de los trabajadores recibieron soluciones IV y el $81,7 \%$ se trató con atropina; ninguno necesito ingresar a la Unidad de Cuidados Intensivos (UCI), y ninguno falleció al egreso. 
Tabla 3. Características relacionadas a la intoxicación de los trabajadores agrícolas intoxicados por pesticidas atendidos en el Hospital de Barranca 2008-2017

\begin{tabular}{|c|c|c|}
\hline Características relacionadas a la intoxicación & $\mathrm{N}=295$ & $\%$ \\
\hline \multicolumn{3}{|c|}{ Tiempo desde la exposición hasta que acudió al hospital } \\
\hline Menor a 1 hora & 92 & 31,2 \\
\hline $1-3 h$ & 159 & 53,9 \\
\hline $4-8 h$ & 38 & 12,9 \\
\hline $9-24 h$ & 6 & 2,0 \\
\hline \multicolumn{3}{|l|}{ Severidad de la intoxicación } \\
\hline Leve & 16 & 5,4 \\
\hline Moderada & 278 & 94,2 \\
\hline Severa & 1 & 0,3 \\
\hline \multicolumn{3}{|l|}{ Manifestación clínica * } \\
\hline Vómitos & 233 & 79,0 \\
\hline Náuseas & 214 & 72,5 \\
\hline Vértigo & 206 & 69,8 \\
\hline Cefalea & 132 & 44,7 \\
\hline Prurito & 72 & 24,4 \\
\hline Visión borrosa & 68 & 23,1 \\
\hline Fiebre & 37 & 12,5 \\
\hline \multicolumn{3}{|l|}{ Tratamiento médico * } \\
\hline Soluciones IV & 294 & 99,7 \\
\hline Atropina & 241 & 81,7 \\
\hline Protectores gástricos & 112 & 38,0 \\
\hline Lavado gástrico & 52 & 17,6 \\
\hline Baño & 1 & 0,3 \\
\hline Protectores hepáticos & 1 & 0,3 \\
\hline \multicolumn{3}{|l|}{ Necesidad de ingreso a UCI } \\
\hline Sí & 0 & 0,0 \\
\hline No & 295 & 100,0 \\
\hline \multicolumn{3}{|l|}{ Complicaciones intrahospitalarias* } \\
\hline Neumonía & 1 & 0,3 \\
\hline Atelectasia & 1 & 0,3 \\
\hline \multicolumn{3}{|l|}{ Estado al egreso } \\
\hline Mejorado & 295 & 100,0 \\
\hline Fallecido & 0 & 0,0 \\
\hline$\left(^{*}\right)$ Respuestas múltiples & & \\
\hline
\end{tabular}

\section{DISCUSIÓN}

En este estudio se buscó determinar las características sociodemográficas y epidemiológicas, relacionadas a la intoxicación por pesticidas de los trabajadores agrícolas atendidos en el Hospital de Barranca (2008-2017). En relación a las características sociodemográficas de los individuos afectados, la mayoría de ellos fueron hombres $(98 \%)$, solteros $(53,6 \%)$ con estudios secundarios culminados $(68,1 \%)$ y se desempeñaban como "aplicador 
de plaguicidas" al momento de la intoxicación $(67,5 \%)$. Se encontró que los más afectados fueron los jóvenes entre los 15 a 25 años (47,5 \%) en comparación con los adultos entre los 46 a 55 años. Díaz et al. ${ }^{(8)}$. reportaron hallazgos semejantes en Totoró, Colombia, donde los cultivadores de papa expuestos a plaguicidas por su trabajo y afectados por los mismos fueron varones $(66,7 \%)$ con edad comprendida entre 15 a 25 años (33,3\%); aunque los trabajadores con mayor afección fueron los que no tenían instrucción $(45,6 \%)$, y solo culminaron primaria (40\%). De igual manera, Cerna y Trigueros encontraron que los pacientes intoxicados por plaguicidas atendidos en el Hospital Nacional San Rafael fueron en su mayoría hombres $(63 \%)$ y solteros $(60 \%){ }^{(9)}$. Para Amador et al. el $62,5 \%$ de los afectados fueron alfabetos ${ }^{(10)}$. En el estudio de Varona et al. hubo mayor frecuencia del sexo masculino, con predominio de agricultores que no tenían escolaridad $(28,5 \%){ }^{(11)}$. Berrio y Mejía reportaron que la población expuesta a plaguicidas en Antioquia fueron aquellos agricultores de 27 a 59 años de edad, donde el $67 \%$ fueron varones.

Respecto a las características epidemiológicas, resalta que la gran mayoría $(97,6 \%)$ no presentó comorbilidades. En relación al IMC, 56,9 \% estaba dentro de los parámetros normales y $32,9 \%$ se encontraba con sobrepeso, lo que es una característica muy importante ya que los organofosforados son de alta liposolubilidad y, por lo tanto, se predice que tendrán un gran volumen de distribución y se distribuirán fácilmente en tejidos y la grasa corporal. Se han observado efectos tóxicos prolongados en pacientes obesos ${ }^{(12)}$.

En relación a las características del pesticida que ocasionó la intoxicación, sobresale que según su clase, tipo y categoría toxica, se trató en su mayoría se trató de un agente fungicida $(39,7 \%)$, organofosforado $(98 \%)$ y medianamente tóxico $(96,3 \%)$, respectivamente. La vía principal de ingreso al organismo fue la respiratoria $(57,3 \%)$ seguido de la piel (42\%). El-Zaemey et al. encontraron resultados opuestos en Yemen, donde el pesticida que causó mayr daño alas mujeres de su estudio fueron los insecticidas $(72,5 \%)$ y los fungicidas $(50,7 \%)^{(13)}$. Mientras que Peña J. encontró en su estudio de intoxicaciones agudas por pesticidas en la región de Arica y Parinacota que los carbamatos fueron los pesticidas que más afectaron a la población de estudio $(44,9 \%)$, seguidos por los organofosforados $(35,4 \%)^{(14)}$. Un panorama distinto presenta Cerna y Trigueros ${ }^{(9)}$. que reportaron una mayor frecuencia de organofosforados (34 \%) y menor proporción de carbamatos (4\%). Por su parte, Janampa encontró mayor presencia de organofosforados $(77,5 \%)$ y menor de carbamatos $(22,5 \%)$. En cuanto a las vías de intoxicación, Pedrozo et al. reportaron que la mayoría de los casos se debieron a exposición por vía oral (80\%) (agua de la red comunitaria contaminada con pesticidas) seguido de la piel $(53,3 \%)$ (6). Estas diferencias pueden deberse a las distintas características sociodemográficas en los estudios. No se encontraron estudios que analizaran la categoría tóxica del pesticida. Se recomienda la realización de nuevos estudios que incluyan esta variable.

En relación a las características de la intoxicación, encontramos que el 53,9 \% de los casos presentó un retraso de 1 a 3 horas en acudir al hospital desde la exposición, el 94,2 \% presentó un nivel moderado de severidad de intoxicación, no hubo necesidad de ingreso a UCl; y prácticamente no presentaron complicaciones intrahospitalarias $(0,7 \%)$. La manifestación clínica más frecuente fue el vómito (79 \%); seguido de náuseas $(72,5 \%)$ y vértigo $(69,8 \%)$. Mientras que las soluciones intravenosas $(99,7 \%)$ y la atropina $(81,7 \%)$ fueron los tratamientos médicos más usados. En comparación, Cerna y Trigueros encontraron que el nivel de severidad de intoxicación más frecuente fue el leve, de igual forma los vómitos o nauseas fueron los síntomas principales, el manejo terapéutico se basó en soluciones intravenosas, lavado gástrico y atropina, y las complicaciones fueron respiratorias, renales y posible daño hepático (13 fallecieron) ${ }^{(9)}$. Pedrozo et al. encontraron que cerca de la mitad de pacientes presentó náuseas, vómitos y cefaleas, unos pocos presentaron prurito y fiebre ${ }^{(6)}$. No se encontraron estudios que analizaran el tiempo en el que el afectado acude al hospital luego de la exposición al pesticida, se recomienda la realización de nuevos estudios que incluyan esta variable.

Es importante indicar que el Código Internacional de Conducta para la Distribución y Utilización de Plaguicidas (FAO) ${ }^{(15)}$, es una herramienta para la prevención de daños causados por el mal uso de los plaguicidas. Este documento aborda puntos como la clasificación de los plaguicidas según su toxicidad; los usos más frecuentes como el control de plagas que afectan los cultivos, el control de las enfermedades transmitidas por vectores y roedores; también los efectos sobre el medio ambiente como la contaminación directa en los cultivos agrícolas, el lavado inadecuado de tanques contenedores, las filtraciones en los depósitos de almacenamiento y los residuos descargados y dispuestos en el suelo; además trata los efectos sobre la salud como la vía de ingreso que puede ser respiratoria, digestiva y dérmica, y la forma de intoxicación (aire inhalado o agua) ${ }^{(16)}$.

Si bien no fue objetivo de nuestra investigación, es importante mencionar la importancia de que los agricultores conozcan los riesgos sanitarios asociados al uso de pesticidas. Jiménez, Pantoja y Ferney hallaron 
un bajo nivel de conocimiento del riesgo de uso de agroquímicos entre la población afectada, la mayoría de los participantes desconocía los posibles efectos que puede desencadenar la exposición a pesticidas (17). Por lo tanto, se recomienda educar e incentivar el uso adecuado de los pesticidas en cuanto a forma y técnica seguras, las cantidades adecuadas, el almacenaje correcto y el equipo de protección necesario para evitar exposiciones nocivas. Una de las funciones del médico ocupacional es preservar y promover la salud de los trabajadores, basado en el conocimiento científico, de la organización y el ambiente de trabajo para así generar las medidas correctivas oportunas. Un ejemplo de ello se observa en Colombia, donde el médico ocupacional participa activamente en los programas de capacitación, seguimiento, vigilancia y control del uso seguro de plaguicidas ${ }^{(18)}$. Asimismo, en Paraguay el médico ocupacional es transcendental, no solo para el manejo clínico de los pacientes, sino como participante en las investigaciones de salud pública relacionadas ${ }^{(6)}$.

Se concluye que los trabajadores agrícolas más afectados por la intoxicación por pesticidas son los varones solteros con grado de instrucción secundaria, con edades comprendidas entre los 15 a 25 años que trabajaban como aplicadores de plaguicidas. Presentaron un IMC normal y no tenían comorbilidades. En relación al pesticida, este era en la mayoría de los casos fungicidas, seguido de los organofosforados, de mediana toxicidad que ingresó al organismo por la vía respiratoria. Dentro de las características relacionadas con la intoxicación, el tiempo entre la exposición la pesticida hasta la llegada al hospital fue de 1 a 3 , y los tratamientos más frecuentes fueron soluciones IV y atropina.

Si bien el presente estudio contribuye a una mejor comprensión de los efectos a corto plazo de la intoxicación por pesticidas en la producción agrícola, también es necesaria la realización de nuevas investigaciones que estimen los efectos de salud a largo plazo de la exposición a pesticidas. Entre estos efectos se incluyen el cáncer, la enfermedad de Parkinson y la enfermedad de Alzheimer. Con este tipo de hallazgos el gobierno deberá revisar la política relacionada al tema para reducir la producción y aplicación de pesticidas. Adicionalmente, como otro mecanismo de prevención se sugiere elaborar estrategias de capacitación dirigidas a agricultores sobre el uso de protectores y la forma adecuada del manejo de los pesticidas de acuerdo a ley; además, sensibilizar respecto al peligro de su empleo, tanto para el ambiente como para la salud personal. Por otro lado, para llevar a cabo mejores prácticas en el manejo clínico de los pacientes intoxicados por pesticidas debe promoverse la realización de pasantías a nivel nacional, en instituciones que manejen el tema de intoxicaciones, tales como el Centro de Información, Control Toxicológico y Apoyo a la Gestión Ambiental (CICOTOX) de la Facultad de Farmacia y Bioquímica de la Universidad Nacional Mayor de San Marcos, y la Unidad de Toxicología Clínica UNITOX del departamento de Emergencia del Hospital Nacional Guillermo Almenara Irigoyen.

\section{REFERENCIAS BIBLIOGRÁFICAS}

1. Maksymiv I. Pesticides: benefits and hazards. J Vasyl Stefanyk Precarpathian National University. 2015; 2(1): p. 70-76.

2. Damalas CA, Eleftherohorinos IG. Pesticide Exposure, safety Issues, and risk assessment indicators. Int J Environ Res Public Health. 2011; 8(5): p. 1402-1419.

3. Vander $\mathrm{H}$. UN human rights experts call for global treaty to regulate dangerous pesticides [internet]. UN News. 2017 [citado 7 Mayo 2017]. Disponible en: https://news.un.org/ en/story/2017/03/552872-un-human-rights-experts-callglobal-treaty-regulate-dangerous-pesticides.

4. Plazas D, Olarte M. Intoxicación por inhibidores de la colinesterasa (organofosforados y carbamatos) en niños y adolescentes: revisión de la literatura y guía de manejo. Acta Colombiana de Cuidado Intensivo. 2011; 1: p. 26-33.

5. Pardo Abdala LM, Pérez Rodríguez S, Gámez Bacallao A. Reportes al Centro Nacional de Toxicología de mujeres en edad fértil expuestas a plaguicidas. Rev Cub Med Mil. 2017; 46(1): 10-8.

6. Pedrozo M, Ocampos S, Galeano R, Ojeda A, Cabello A, De Assis D. Casos de intoxicación aguda por plaguicidas en la colonia Puerto Pirapó, Itapúa, Paraguay, febrero de 2014. Biomédica. 2017 37(1):158-63.

7. Gutiérrez Lesmes OA, Loboa Rodríguez NJ, Plata Casas LI. Situación epidemiológica de la intoxicación por sustancias químicas en el departamento del Meta-Colombia, periodo 2009-2014. Biosalud. 201716(1):30-42.

8. Diaz S, Sánchez F, Varona M, Eljach V, Muñoz N. Niveles de colinesterasa en cultivadores de papa expuestos ocupacionalmente a plaguicidas, Totoró, Cauca. Rev Univ Ind Santander Salud. 2017;49(1):85-92.

9. Cerna Urrutia LE, Trigueros Jovel JR. Epidemiología y evolución clínica de pacientes intoxicados por plaguicidas atendidos en el Hospital Nacional San Rafael en el período de enero 2013 a junio 2014. Tesis doctoral. El Salvador: Universidad Dr. José Matías Delgado; 2015.

10. Amador Rosa ME, González Flores OM, Paz Jiménez HG. Caracterización de intoxicaciones agudas por plaguicidas en el departamento de Intibucá. Rev Med Hondur. 2016; 84(34):92-94.

11. Varona ME, Díaz SM, Briceño L, Sánchez $\mathrm{Cl}$, Torres $\mathrm{CH}$, Palma $\mathrm{RM}$, et al. Determinantes sociales de la intoxicación por plaguicidas entre cultivadores de arroz en Colombia. Rev salud pública. 2016; 18(4): p. 617-629.

12. Lee DH, Jung KY, Choi YH, Cheon Y. Body mass index as a prognostic factor in organophosphate-poisoned patients. Am J Emerg Med. 2014; 32(7):693-6.

13. El-Zaemey S, Fritschi L, Heyworth J. Occupational pesticide exposure among Yemeni women. Environ Res. 122(1):45-51.

14. Peña J. Caracterización de los casos por intoxicaciones agudas por plaguicidas en la región de Arica y Parinacota, período 
Caracterización de la intoxicación ocupacional por pesticidas en trabajadores agrícolas atendidos en el Hospital Barranca Cajatambo 2008 - 2017

2005-2010. [Tesis]. Chile: Universidad de Chile; 2016.

15. FAO. Código Internacional de Conducta para la Distribucion y Utilizacion de Plaguicidas. Roma: Grupo de la FAO; 2013.

16. Del Puerto A, Suárez S, Palacio D. Efectos de los plaguicidas sobre el ambiente y la salud. Rev Cubana Hig Epidemiol 2014; 52(3): p. 372-387.

17. Jimenez C, Pantoja A, Ferney H. Riesgos en la salud de agricultores por uso y manejo de plaguicidas, microcuenca "La Pila". Univ Salud 2016; 18(3):417-31.

18. López K, Villamil D, Zambrano MF. Diagnóstico ocupacional de las condiciones de trabajo en unas poblaciones agrícolas expuestas a plaguicidas organofosforados. Rev CES Salud Pública. 2016; 7(1):17-24.

Fuentes de financiamiento:

Este artículo ha sido financiado por los autores.

Conflictos de interés:

Los autores declaran no tener ningún conflicto de interés.

\section{Correspondencia:}

Lincol Marx Cruz Aquino

Dirección: Calle Elcorrobarrutia N 232 - Distrito Huacho, Provincia Huaura, Departamento Lima

Teléfono: 964806510

Correo electrónico: lincolcruz@gmail.com

\section{Recibido: 05 de octubre de 2018}

Evaluado: 20 de noviembre de 2018

Aprobado: 28 de noviembre de 2018

๑ La revista. Publicado por Universidad de San Martín de Porres, Perú. (cc) Br Licencia de Creative Commons Artículo en acceso abierto bajo términos de Licencia Creative Commons Atribución 4.0 Internacional. (http://creativecommons.org/licenses/by/4.0/)

ORCID iDs

Lincol Marx Cruz Aquino Maritza Dorila Placencia Medina

https: //orcid.org/0000-0002-3347-0119 https://orcid.org/0000-0003-3624-3461 